\title{
Is There a Role for Liver Transplantation in Non-Colorectal Liver Metastases?
}

\author{
Uta Herden Björn Nashan \\ Department of Hepatobiliary Surgery and Transplantation, University Medical Center Hamburg-Eppendorf, Hamburg, Germany
}

\section{Keywords}

Liver metastases - Liver transplantation .

Neuroendocrine tumor

\section{Summary}

Due to the high blood flow, especially blood from the intestinal tract via the portal vein, the liver is a preferred organ for metastases. In case of advanced, irresectable liver metastases liver transplantation (LTX) remains an attractive option. However, due to high recurrence rates or a lack of data, up to date, metastases from neuroendocrine tumors (NETs) are the only accepted indication for LTX in non-colorectal liver metastases. In this regard, LTX is only justified in patients in which complete tumor resection (R0 resection) of the NET is achievable. A literature review revealed no clear patient selection criteria but transplantation should definitively achieve an $\mathrm{R} 0$ resection with complete freedom of tumor. The available data regarding the outcome following LTX for NETs show a comparable short- and long-term outcome for patients transplanted for other malignancies, e.g. hepatocellular carcinoma, or also benign indications in the high MELD (model for end-stage liver disease) era. Thus, most data prove a better post-transplant outcome and a lower recurrence rate in patients with a good differentiation of the tumor, a low proliferation index (Ki67), and a portal drainage of the NET.

(c) 2015 S. Karger GmbH, Freiburg

\section{Introduction / Liver Transplantation in Hepatic Malignancies}

Liver transplantation (LTX) as a treatment option for hepatic malignancies to achieve an $\mathrm{R} 0$ resection in otherwise irresectable tumors evolved into a standard procedure concerning selected tumor entities.

In 1996, Mazzaferro et al. [1] showed an excellent overall and recurrence-free survival in patients undergoing LTX for small hepatocellular carcinomas (HCC) within the so-called 'Milan criteria' (singular $\mathrm{HCC} \leq 5 \mathrm{~cm}$ or up to three $\mathrm{HCC} \leq 3 \mathrm{~cm}$ without macrovascular invasion). Also, extension of the HCC criteria such as the 'University of California San Francisco (UCSF) criteria' (single tumor $\leq 6.5 \mathrm{~cm}$ or up to three tumors $\leq 4.5 \mathrm{~cm}$ with a total tumor volume $\leq 8 \mathrm{~cm}$ ) or the 'up-to-seven criteria' (sum of the number of nodules and diameter of the largest tumor in $\mathrm{cm}$ ) showed a comparable and good outcome $[2,3]$. Therefore, up to date, HCC is a standard indication for LTX with an outcome comparable to patients transplanted for benign indications.

Early results of LTX in patients with non-resectable cholangiocellular carcinoma showed a high recurrence rate with a resulting poor patient survival [4-6]. Recently, the Mayo group could show an excellent overall survival (1-/5-year survival rate $92 \% / 82 \%)$ and a low recurrence rate (1-/5-year recurrence rate $0 \% / 12 \%)$ in highly selected patients (small tumor $<3 \mathrm{~cm}$, no lymph node or distant metastases) with a stringent protocol including neoadjuvant radiochemotherapy [7]. However, a disadvantage of this study consists in an absence of malignancy in the histopathological result in $42 \%$ of the explanted livers. Further studies are needed and ongoing to verify the benefit of LTX in patients with cholangiocellular carcinoma.

Another primary liver malignancy which has evolved into a standard indication for LTX is non-resectable hepatoblastoma in children, in selected cases even in the situation of controlled distant metastases [8].

\section{KARGER \\ Fax +497614520714

PD Dr. med. Uta Herden 
Table 1. Overview about existing studies concerning LTX for NET - with 1-, 3-, 5-year survival and disease-free survival rates

\begin{tabular}{|c|c|c|c|c|c|c|c|}
\hline \multirow[t]{2}{*}{ Author } & \multirow{2}{*}{$\begin{array}{l}\text { Patients, } \\
\mathrm{n}\end{array}$} & \multicolumn{3}{|c|}{ Overall survival, \% } & \multicolumn{3}{|c|}{ Disease-free survival, \% } \\
\hline & & 1 year & 3 years & 5 years & 1 year & 3 years & 5 years \\
\hline Le Treut et al. [13] & 213 & 81 & 65 & 52 & 65 & 40 & 30 \\
\hline Gedaly et al. [14] & 150 & 81 & 65 & 49 & 77 & 50 & 32 \\
\hline Mazzaferro et al. [15] & 24 & NR & NR & 90 & NR & NR & 77 \\
\hline
\end{tabular}

NR $=$ Not reported
Instead of primary liver malignancies, the liver is a preferred organ for metastases of different primary tumors, especially from the intestine. In general, metastatic diseases represent a contraindication for organ transplantation. There are only few data regarding the outcome following LTX for irresectable colorectal metastases. The SECA study in Norway [9], an open, prospective pilot study including 21 patients with non-resectable liver-only colorectal liver metastases, analyzed the outcome following LTX. Only patients fulfilling strict inclusion criteria (e.g. radical resection of the primary tumor, extensive pre-transplant staging including laparotomy with lymphadenectomy in the hepatoduodenal ligament and negative frozen section examination, minimum of 6 weeks of chemotherapy pre-transplant, good performance status (ECOG 0 or 1)) were included in the study. The recurrence rate after LTX was very high, with diagnosis of a local or metastatic recurrence in 19 of 21 patients after a median time of 6 months. The patient survival rates were 95,68 , and $60 \%$ at 1,3 , and 5 years after LTX, respectively. Overall, due to the existing organ shortage in almost all countries and the comparable worse outcome following LTX for colorectal liver metastases, it is widely recognized as a contraindication for transplantation.

Regarding LTX for non-colorectal liver metastases, the only remaining accepted indication is metastases from neuroendocrine tumors (NETs). NETs originate from the widespread neuroendocrine system with primary localization mostly in the gastrointestinal tract, followed by the pancreas, lung, kidney, ovaries, and other sites. The liver is the most common metastatic site, and in most cases liver metastases are already present at the time of diagnosis [10]. Resection of the primary tumor and the metastases remains the best therapeutic option. Despite of the concept of cytoreductive surgery in NETs being incorporated in a multimodal therapy regime, resection should be able to remove the bulk of the liver metastases [11]. Therefore, LTX seems to be a possible option in selected cases with non-resectable liver metastases. A review of the literature reveals a number of small studies and meta-analyses regarding the subject of LTX for NET liver metastases.

\section{Key Questions in LTX for NETs}

- What is the outcome regarding patient survival and tumor recurrence in patients transplanted for NET liver metastases?

- Which selection criteria should be used to select patients for LTX?

- What is the best time point for transplantation?
Table 2. Milan criteria for LTX in patients with hepatic metastasis of a NET [15]

\section{Inclusion criteria}

1. Confirmed histology of carcinoid tumor (low-grade neuroendocrine tumors) with or without syndrome

2. Primary tumor drained by portal system (pancreas and intermediate gut: from distal stomach to sigmoid colon) removed with a curative resection (pre-transplant removal of all extrahepatic tumor deposits) through surgical procedures different and separate from transplantation

3. Metastatic diffusion to liver parenchyma $\leq 50 \%$

4. Good response or stable disease for at least 6 months during pre-transplant period

5. Age $\leq 55$ years

\section{Exclusion criteria}

1. Small-cell carcinoma and high-grade neuroendocrine carcinomas (noncarcinoid tumors)

2. Other medical/surgical conditions contraindicating liver transplantation, including previous tumor

3. Non-gastrointestinal carcinoids or tumors not drained by the portal system

Fan et al. [12] performed a literature review regarding the aspect of LTX for NET liver metastases. They found 46 relevant publications including a total of 873 patients undergoing LTX for NETs. Studies include prospective and retrospective comparative cohort studies and case-controlled studies; no prospective randomized trial was available for this topic. The reported overall 5 -year posttransplant survival rates varied between 47 and $90 \%$. One of the largest included studies analyzing the outcome following LTX for NETs in Europe (based on LTX data of 213 patients from 35 centers in 11 European countries) showed an overall 1-, 2-, 3-, 4-, and 5 -year survival of $81,73,65,55$, and $52 \%$, respectively [13]. Another large study $(\mathrm{n}=150)$ analyzing the UNOS data found 1-, 3-, and 5 -year overall survival rates of 81,65 , and $49 \%$, respectively [14]. Overall, the reported survival rates are comparable to patients undergoing LTX for HCC within the generally used thresholds and are likewise comparable to patients transplanted for non-malignant indications within the MELD (model for end-stage liver disease) allocation era. The described 5-year recurrence-free survival varied in the different studies from 18 to $77 \%$ [12]. In the analysis of the Europe Liver Transplant Registry, Le Treut et al. [13] found recurrence-free survival rates of $65,49,40,33$, and $30 \%$ at $1,2,3,4$, and 5 years after LTX, respectively, in the previously mentioned 213 patients. The analysis of the UNOS database showed 1-, 3-, and 5 -year recurrence-free survival rates of 77,50 , and $32 \%$, respectively [14]. An overview about the existing studies with post-transplant survival and disease-free survival rates is given in table 1. 


\section{Guidelines for Neuroendocrine Tumors (NET)}

A review of the literature supports that candidates with NET are expected to have a low risk of waiting list drop-out. Initial recommendations included age less than 60. Older patients with a lot of disease burden may be referred to transplant as a last resort, leading to poor outcomes, while data presented at the AASLD show that very young patients with NET and early stage disease do well. Committee members believed that these initial guidelines could include strict criteria that could be expanded based upon the experience of the RRBs.

\section{Transplant programs should also be aware of these criteria when submitting exceptions for NET. RRBs should consider the following criteria when reviewing exception applications for candidates with NET.}

1. Recipient age $<60$ years.

2. Resection of primary malignancy and extra-hepatic disease without any evidence of recurrence at least six months prior to MELD exception request.

3. Neuroendocrine Liver Metastasis (NLM) limited to the liver, Bi-lobar, not amenable to resection.

4. Tumors in the liver should meet the following radiographic characteristics:

- a. CT Scan: Triple phase contrast

- i. Lesions may be seen on only one of the three phases

$\circ \quad$ ii. Arterial phase: may demonstrate a strong enhancement

- iii. Large lesions can become necrotic/calcified

- b. MRI Appearance:

○ i. Liver metastasis are hypodense on T1 and hypervascular in T2 wave images

- ii. Diffusion restriction

- iii. Majority of lesions are hypervascular on arterial phase with wash -out during portal venous phase

○ iv. Hepatobiliary phase post Gadoxetate Disodium (Eovist): Hypointense lesions are characteristics of NET

5. Consider for exception only those with a NET of Gastro-entero-pancreatic (GEP) origin tumors with portal system drainage. Note: Neuroendocrine tumors with the primary located in the lower rectum, esophagus, lung, adrenal gland and thyroid are not candidates for automatic MELD exception.

6. Lower - intermediate grade following the WHO classification. Only well differentiated (Low grade, G1) and moderately differentiated (intermediate grade G2). Mitotic rate $<20$ per $10 \mathrm{HPF}$ with less than $20 \%$ ki 67 positive markers.

7. Tumor metastatic replacement should not exceed $50 \%$ of the total liver volume

8. Negative metastatic workup should include one of the following:

- a. Positron emission tomography (PET scan)

- b. Somatostatin receptor scintigraphy

- c. Gallium-68 (68Ga) labeled somatostatin analogue 1,4,7,10tetraazacyclododedcane-N, N', N",N'"-tetraacetic acid (DOTA)-D-Phe1-Try3octreotide (DOTATOC), or other scintigraphy to rule out extra-hepatic disease, especially bone metastasis.

Note: Exploratory laparotomy and or laparoscopy is not required prior to MELD exception request.

9. No evidence for extra-hepatic tumor recurrence based on metastatic radiologic workup at least 3 months prior to MELD exception request (submit date).

Fig. 1. Recommendations from the OPTN to select patients with NET for LTX [20].

10. Recheck metastatic workup every 3 months for MELD exception increase consideration by the Regional Review Board. Occurrence of extra-hepatic progression - for instance lymph-nodal Ga68 positive locations - should indicate de-listing. Patients may come back to the list if any extra-hepatic disease is zeroed and remained so for at least 6 months.

11. Presence of extra-hepatic solid organ metastases (i.e. lungs, bones) should be a permanent exclusion criteria

Mazzaferro, who had already defined the 'Milan criteria' as selection criteria for patients with HCC undergoing LTX, described in his work 'Neuroendocrine tumors metastatic to the liver: how to select patients for liver transplantation?' [15] possible selection criteria for patients with NETs and LTX based on a literature review (1970-2006). Table 2 shows the detailed inclusion and exclusion criteria for transplantation in patients with neuroendocrine liver metastases analogously to his publication.

Different publications showed a reduced post-transplant survival in patients transplanted for poorly differentiated NETs compared to well-differentiated NETs. Ki67 as proliferation index gives further information about the differentiation of the NET. In gen- 
eral, a Ki67 value $<10 \%$ characterizes a well-differentiated tumor and select patients for LTX [15-17]. Studies regarding the benefit of a further reduction of the Ki67 value (e.g. $<2 \%$ ) mostly failed to prove a statistically significant benefit in post-transplant patient survival or recurrence reduction $[18,19]$.

The consideration to perform LTX only in NETs with portal drainage is based on the idea that solely in these cases the liver represents the first metastatic station and that, accordingly, no further tumor spread exists. This means that only gastrointestinal NETs from the distal stomach to the middle colon and NETs from the pancreas should be considered for LTX.

In every case the primary tumor site should be resected prior to LTX in a curative intent, including complete tumor resection and locoregional as well as distant lymph node dissection, thus achieving an R0 resection. Applying the strict selection criteria given in table 2, Mazzaferro et al. [15] described an excellent post-transplant 5-year survival of $90 \%$ and a 5 -year recurrence-free survival of $77 \%$ in a prospective series $(n=24)$. However, other groups also showed good outcomes in patients with extended criteria concerning patient age, tumor mass, or primary tumor site [17, 19].

Due to the good outcome of individual patients undergoing LTX for NETs, the Organ Procurement and Transplantation Network (OPTN) published recommendations for selecting patients in the United States for LTX with NETs. Selection criteria include recipient age $<60$ years, limitation of the NET metastases to the liver with a bilobar involvement and not amenable to resection, and resection of the primary malignancy without evidence for recurrence for at least 6 months. Only patients with well to moderately differentiated NETs (G1/G2) with a low mitotic rate $(<20$ per 10 high power fields) and a Ki67 value $<20 \%$ which originate from the gastroenteropancreatic system with portal system drainage should undergo LTX. Furthermore, the guidelines contain instructions for radiological workup including time interval, type of diagnostics, and radiographic characteristics. The detailed recommendations are shown in figure 1 [20].
Overall, NETs are mostly slow-growing tumors with long periods of stable disease. There are controversial opinions regarding the best time point of LTX in patients with NET liver metastases. Whereas Mazzaferro et al. [15] postulate a good response or a stable disease for at least 6 months prior to LTX, many experts support that asymptomatic patients with stable disease do not require LTX, whereas LTX should be performed in case of non-resectable liver metastases under progress from refractory to non-medical treatment [12]. However, different studies found a better outcome in patients with a longer waiting time [14].

\section{Conclusion}

In summary, NETs remain the only tumor entity in which LTX is justifiable and indicated in case of irresectable liver metastases. Literature review reveals no clear patient selection criteria; however, transplantation should definitively achieve an R0 resection with complete freedom of tumor. As expected, most data proved a better post-transplant outcome and a lower recurrence rate in patients with a good differentiation of the tumor and a low proliferation index (Ki67 < 10\%). Additionally, an outcome benefit is postulated in patients with NETs with a portal drainage.

Overall, the actually reported short- and long-term outcome of LTX in these selected patients with NET liver metastases is comparable to patients transplanted for other malignancies, e.g. HCC, or benign indications.

\section{Disclosure Statement}

UH: Travel expenses from Astellas, Novartis, Teva, and BMS. BN: Research support from Novartis; travel expenses and/or speaker's fees from Astellas, Bayer, Genzyme/Sanofi, and Novartis; clinical studies: Alexion, BMS, and Novartis.

\section{References}

1 Mazzaferro V, Regalia E, Doci R, Andreola S, Pulvirenti A, Bozzetti F, Montalto F, Ammatuna M Morabito A, Gennari L: Liver transplantation for the treatment of small hepatocellular carcinomas in patients with cirrhosis. N Engl J Med 1996;334:693-699.

2 Yao FY, Ferrell L, Bass NM, Watson JJ, Bacchetti P, Venook A, Ascher NL, Roberts JP: Liver transplantation for hepatocellular carcinoma: expansion of the tumor size limits does not adversely impact survival. Hepatology 2001;33:1394-1403.

3 Mazzaferro V, Llovet JM, Miceli R, et al: Predicting survival after liver transplantation in patients with hepatocellular carcinoma beyond the Milan criteria: a retrospective, exploratory analysis. Lancet Oncol 2009; 10:35-43.

4 O'Grady JG: Treatment options for other hepatic malignancies. Liver Transpl 2000;6(suppl 2):S23-29.
Casavilla FA, Marsh JW, Iwatsuki S, Todo S, Lee RG, Madariaga JR, Pinna A, Dvorchik I, Fung JJ, Starzl TE: Hepatic resection and transplantation for peripheral cholangiocarcinoma. J Am Coll Surg 1997;185:429-436.

6 Ringe B, Wittekind C, Bechstein WO, Bunzendahl H, Pichlmayr R: The role of liver transplantation in hepatobiliary malignancy. A retrospective analysis of 95 patients with particular regard to tumor stage and recurrence. Ann Surg 1989;209:88-98.

7 Rea DJ, Heimbach JK, Rosen CB, Haddock MG, Alberts SR, Kremers WK, Gores GJ, Nagorney DM: Liver transplantation with neoadjuvant chemoradiation is more effective than resection for hilar cholangiocarcinoma. Ann Surg 2005;242:451-458; discussion 458461.

8 Meyers RL, Tiao GM, Dunn SP, Langham MR Jr: Liver transplantation in the management of unresectable hepatoblastoma in children. Front Biosci (Elite Ed) 2012;4:1293-1302.
Hagness M, Foss A, Line PD, et al: Liver transplantation for nonresectable liver metastases from colorectal cancer. Ann Surg 2013;257:800-806.

10 Alagusundaramoorthy SS, Gedaly R: Role of surgery and transplantation in the treatment of hepatic metastases from neuroendocrine tumor. World J Gastroenterol 2014;20:14348-14358.

11 Que FG, Sarmiento JM, Nagorney DM: Hepatic surgery for metastatic gastrointestinal neuroendocrine tumors. Cancer Control 2002;9:67-79.

12 Fan ST, Le Treut YP, Mazzaferro V, Burroughs AK, Olausson M, Breitenstein S, Frilling A: Liver transplantation for neuroendocrine tumour liver metastases. HPB (Oxford) 2015;17:23-28.

13 Le Treut YP, Gregoire E, Klempnauer J, et al: Liver transplantation for neuroendocrine tumors in Europe - results and trends in patient selection: a 213-case European liver transplant registry study. Ann Surg 2013; 257:807-815. 
14 Gedaly R, Daily MF, Davenport D, McHugh PP, Koch A, Angulo P, Hundley JC: Liver transplantation for the treatment of liver metastases from neuroendocrine tumors: an analysis of the UNOS database. Arch Surg 2011;146:953-958.

15 Mazzaferro V, Pulvirenti A, Coppa J: Neuroendocrine tumors metastatic to the liver: how to select patients for liver transplantation? J Hepatol 2007;47:460-466.

-16 Ahlman H, Friman S, Cahlin C, Nilsson O, Jansson S, Wangberg B, Olausson M: Liver transplantation for treatment of metastatic neuroendocrine tumors. Ann N Y Acad Sci 2004;1014:265-269.
17 Rosenau J, Bahr MJ, von Wasielewski R, Mengel M, Schmidt HH, Nashan B, Lang H, Klempnauer J, Manns MP, Boeker KH: Ki67, E-cadherin, and p53 as prognostic indicators of long-term outcome after liver transplantation for metastatic neuroendocrine tumors. Transplantation 2002;73:386-394.

18 van Vilsteren FG, Baskin-Bey ES, Nagorney DM, Sanderson SO, Kremers WK, Rosen CB, Gores GJ, Hobday TJ: Liver transplantation for gastroenteropancreatic neuroendocrine cancers: defining selection criteria to improve survival. Liver Transpl 2006;12:448456
9 Olausson M, Friman S, Herlenius G, Cahlin C, Nilsson O, Jansson S, Wangberg B, Ahlman H: Orthotopic liver or multivisceral transplantation as treatment of metastatic neuroendocrine tumors. Liver Transpl 2007; 13:327-333.

20 Organ Procurement and Transplantation Network (OPTN): Guidance on MELD PELD exception review. June 2, 2015. http://optn.transplant.hrsa.gov/resources/ by-organ/liver-intestine/guidance-on-meld-peld-exception-review/\#PSC. 